$\xi=-1$

\title{
Analysis of QoS for PAT and EnOPATr System in WSN
}

\author{
Devare Avinash $\mathrm{S}^{1 *}$, G Krishna Mohan ${ }^{2}$, \\ ${ }^{1,2}$ Koneru Lakshmaiah Education Foundation, \\ Vaddeswaram, Guntur, Andhra Pradesh, India - 522502 \\ *Email: devarea9@gmail.com,
}

\begin{abstract}
In the situations of natural disasters, Wireless Sensor Networks (WSN) is extensively used. Quality of Service (QoS) is an important parameter for ensuring effectiveness and robustness of service provided. QoS gets degraded when being applied in various applications of WSN. Qos expected in WSN applications gets reduced further. In this case, efficient use of the scarce resources is important to ensure continuous data transmission is ensured by proficient use of resources which are limited resources. There are many WSN protocols which focus on broadcasting of important information. But there are many parameters which get neglected by these protocols. For instance, normal data traffic gets neglected by these protocols. Hence, this research proposes EnOPATr system which is independent and distributed. The essential and normal data is handled at the same time in EnOPATr which achieves dependable and congestion free data. In a sensor node, power consumption can be reduced by decreasing the rate of packet retransmission. This is because of congestion in network. The simulation of the proposed system is done using NS-2 software which shows that the proposed system is able to reduce network congestion thereby improving the performance..
\end{abstract}

Keywords: Wireless Sensor Network, Congested control, Packet Discarding, PAT, EnOPATr

\section{Introduction}

Wireless Sensor Network (WSN) consists is a collection of hundreds or thousands of sensor nodes. WSN is used to provide various features such as remote monitoring of services, and specific operations in conditions of hazardous nature [1]. The conditions specifically are such that the involvement of human being could be risky. All the necessary and important information is collected related to occurrence of certain specific events. As shown in figure-1, WSN makes use of data centric approach. The central node accepts the data from various nodes and then transmits to the gateway for sending it to end user. This approach suffers from limitation in that each sensor node in WSN has scarce resource for producing huge amount of data. This work tries to reduce the underlining congestion in the WSN network for the specific situations of scare resources [8].

The scope of the research is focusing on Enhancement of Path Assured Transport Protocol (EnOPATr) to solve Congestion in WSN. The rest of this paper is organized as follows. Section II reviews the literature related to congested problem. Section III proposes the EnOPATr system. It includes the review of the proposed algorithm. Section IV presents the result of the experiment. The paper finishes with the concluding remarks.

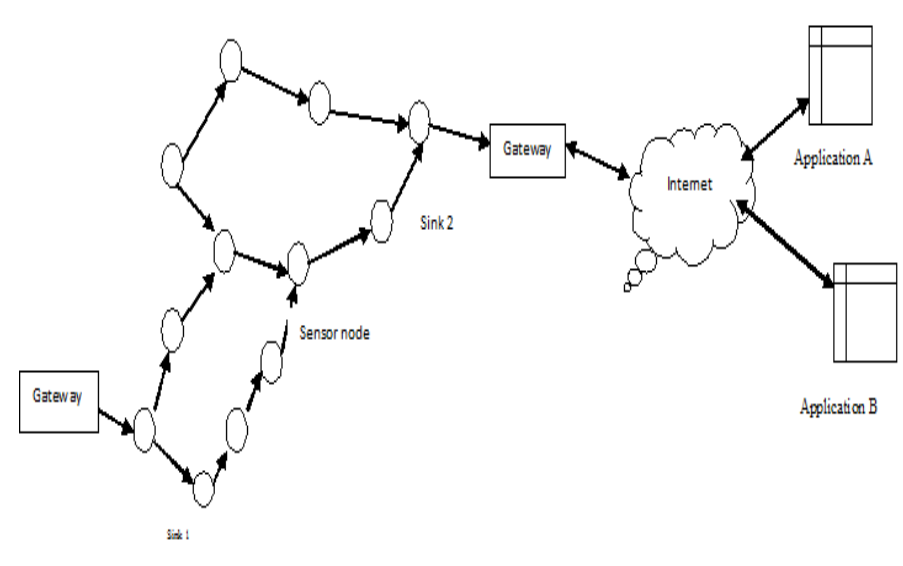

Fig 1: WSN architecture

\section{Previous Work Done}

A limited power and resources are the limitations of WSN. This is clear from the previous work. So, the current review focuses on urgent as well as normal data.

\section{A. The Congestion Problem}


Congestion is caused due to limited processing power and resource utilization of nodes in WSN. When the data being sent is not in sync with receiver node, this leads to congestion also. This is not energy efficient for the node. The node gets overloaded due to limited size of memory. The energy of the node gets wasted due to WSN. Limited bandwidth and

capacity of node, leads to congestion in the network. The data packets are more than what a node can receive. Due to this overloading, the fairness in the traffic is not achieved. Data therefore will affect the fairness of the traffic due the Large number of data needed to be transmitted with limited resource provided $[2,3]$.

There are two factors which play a major role in congestion control. These are memory of sensor node notification and packet traveling methodology which is used for congestion control scheme. However, for urgent situations, more packets get transferred in short time span. This is needed for getting the information regarding the event as fast as possible. For preventing congestion control within WSN, there are many protocols. Out of these protocols, there are few protocols focus on critical data transmission $[9,10]$.

\section{A. A protocol for Urgent information transmission}

It is reliable (RETP-UI) RETP-UI does classify different traffics into three main categories. For each of such categories, different queue is maintained. The total length of queue and its overall fluctuations in the network determines the congestion in network RETP-UI has additional benefits of increased performance. It also gives lesser time for the packet to be received. Hence, the overall packet loss is reduced $[6,7]$.

\section{B. Protocol Transfer of Data with Assured Way of Path}

The given protocol has three varied procedures. The first step involves data node initiating a request which is blocking in nature to node for blocking their regular data transmission. As a result of the blocking mechanism, the path of data transmission gets cleared. In the second step, protocol exchanges the important data to sink node that is also known as master node. The protocol then gets a reply from the sink node in the same duration. Upon completion of the data transmission, the master or the sink node sends the release message. The path hence created, creates the provision for data to the transmitted collision free and hence reduce the delay. However, this protocol is applicable only to critical data and normal data $[4,11]$.

\section{The Proposed System and Workflow}

There are various protocols made exclusively for urgent data transmission. In PAT protocol, sink node receives request from the sensor node for transmission of data. The current systems, working on this protocol focus only on sending the urgent data. The proposed work differs from the existing work, by sending both urgent as well as normal data. In the previous systems, the sensor node receives only the high priority data from sink node. This is explained diagrammatically in Figure-2. As shown in the figure-2, the high priority data is sent first while the normal data traffic is blocked. Congestion in the network gets overcome by this approach

Simultaneously, sensor nodes produce normal data at other locations. Sensor node cannot sent data in the network due to this blocking of network. [12,13].

As the sensor node has limited computation power in terms of memory, what gets generated at this node is a normal data packet.
The less memory within the sensor node prevents storage of data. The normal data packet gets generated at the sensor node which does not get stored due to less memory. The current work has there are three phase Sink node identifies in the network if urgent data is there or not. If yes, the UREQ gets dispatched from sink node. In case the sensor node has a urgent data, it dispatches UREQ to the sink node.

UREQ gets propagated to the sink node by a number of hops. Along the way, some information is added by the nodes which are in-between the sink node. The UREQ request will come to the sink node by a number of hops on intermediate sensor nodes. Various additional information get added by the intermediate node to the request packet such as their ID. Upon receipt of the requested packet, the sink node will immediately issue a broadcast request which will have the ID information of the intermediate node. The intermediate nodes are the one which had received the requested packet. When the rest of the nodes receive the blocking request, the sink node checks the neighbor ID with the ID present in the blocking request. In case the two ID's match, the sink node will stall the normal data traffic, otherwise traffic will be redirected to the sink node. Sensor node receives from sink node various blocking requests. Each of the nodes near to the sink node will sent Information as another secondary sink node. When sink node received this information contained in the packet, selection of nodes is done. One of them acts as the backup /secondary sink node. At a time, normal and urgent data is generated. The normal data gets transferred to the backup or secondary sink node. The urgent data gets transferred to the original sink node. Upon completion of critical data being sent, the original sink node communicates to all nodes the block release request. After this request gets received at backup/secondary sink node, it will initiate the process dispatching normal data which is a compilation of normal data generator node [5].

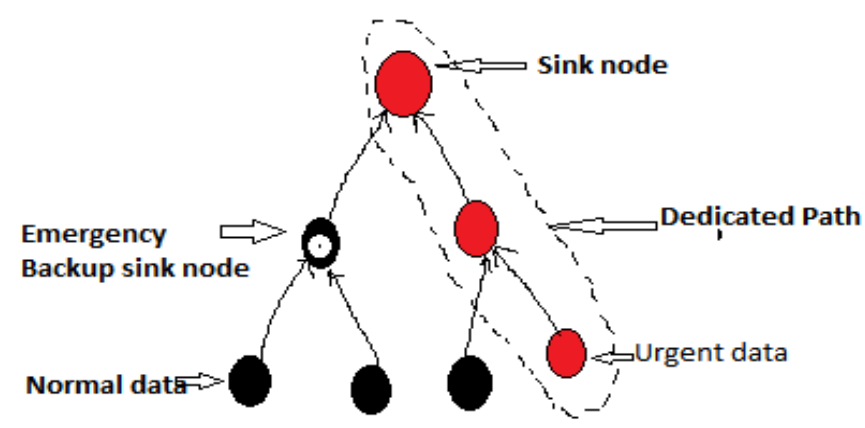

Fig 2: Proposed system EnOPATr

The simulation is conducted using Network Simulator 2 (NS-2) simulator which contains huge resources for conducting simulation of WSN environments. NS 2 gives the needed resources for simulation of various WSN protocols.NS2 also provides a very discrete environment for conducting WSN research.

\section{Result and Discussion}

In order to evaluate PAT vs. EnOPATr performance, we measure the parameters in two different scenarios: normal data transfer and urgent data transfer. Interval, node, packet size wise are the parameters used in evaluation of the PAT and EnOPATr system.

\section{A.Interval}


Figure-3 provides the interval vs. packet duration ratio in PAT and EnOPATr system. The black line shows more packet delivery ratio as compared to red line. Black line indicates EnOPATr protocol and red line indicates PAT protocol.

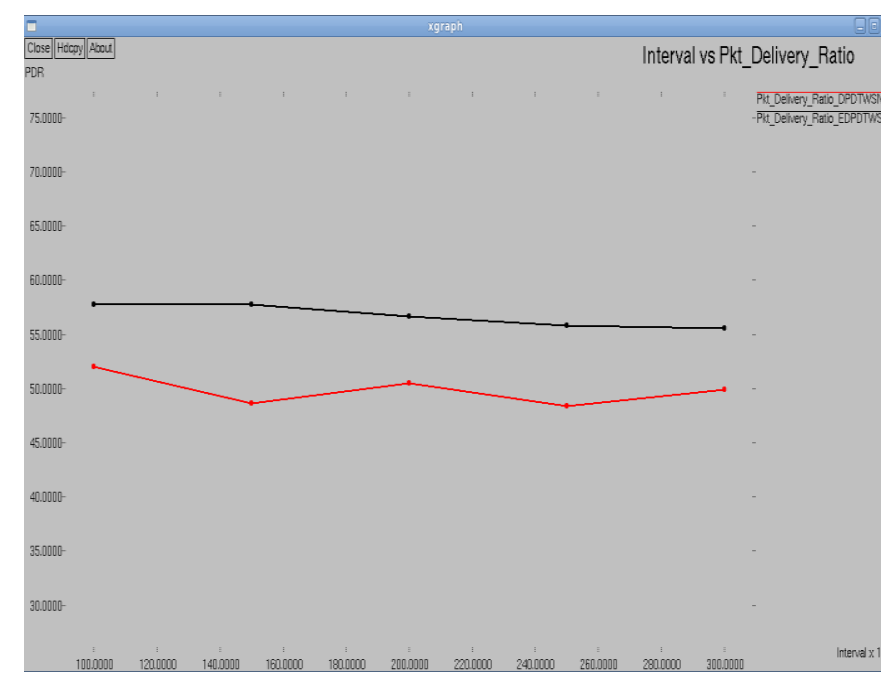

Fig 3: Graph Shows Interval Vs Packet Delivery Ratio

Figure-4 shows the interval vs. delay duration ratio in PAT and EnOPATr system. The delay is more in PAT system as compared to EnOPATr. Delay in the network is hence avoided. QoS automatically decreases due to delay in network.

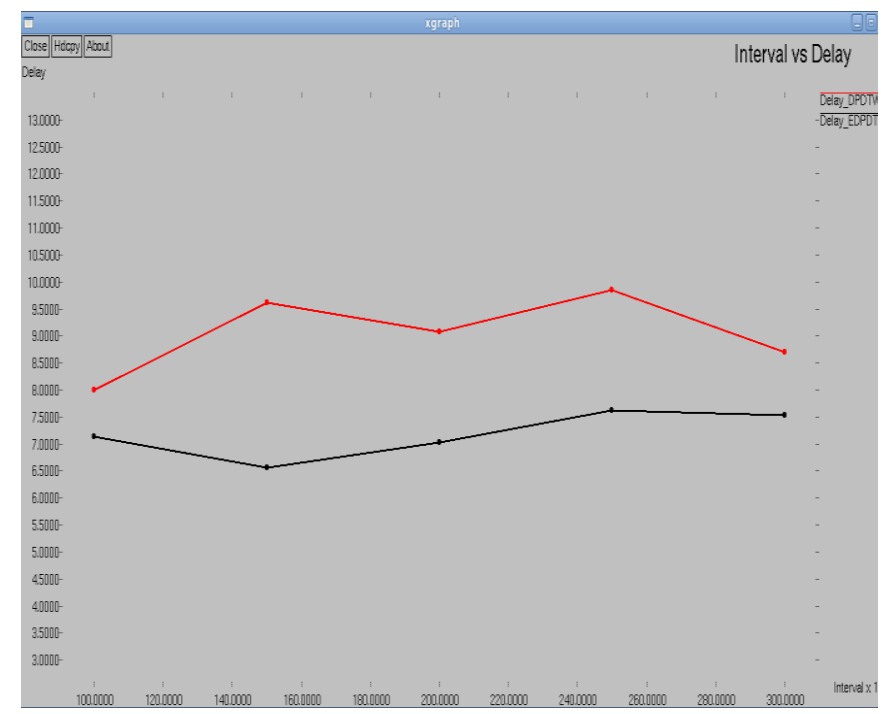

Fig 4: Graph Shows Interval Vs Delay

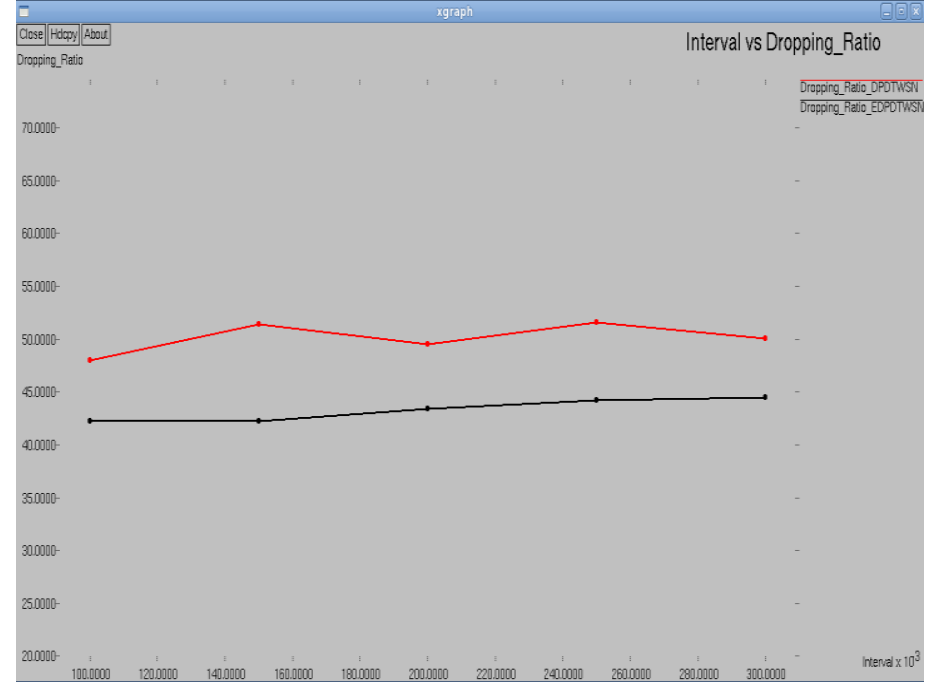

Fig 5: Graph Shows Interval Vs Packet Dropping Ratio

Figure-5 shows the interval vs. packet dropping ratio in PAT and EnOPATr system. The packet dropping is more in PAT system as compared to EnOPATr.

\section{B.Node}

Figure-6 shows node vs. packet delivery ratio in PAT and EnOPATr system. The packet successfully reach to destination is more in EnOPATr as compared to PAT system with different node scenario.

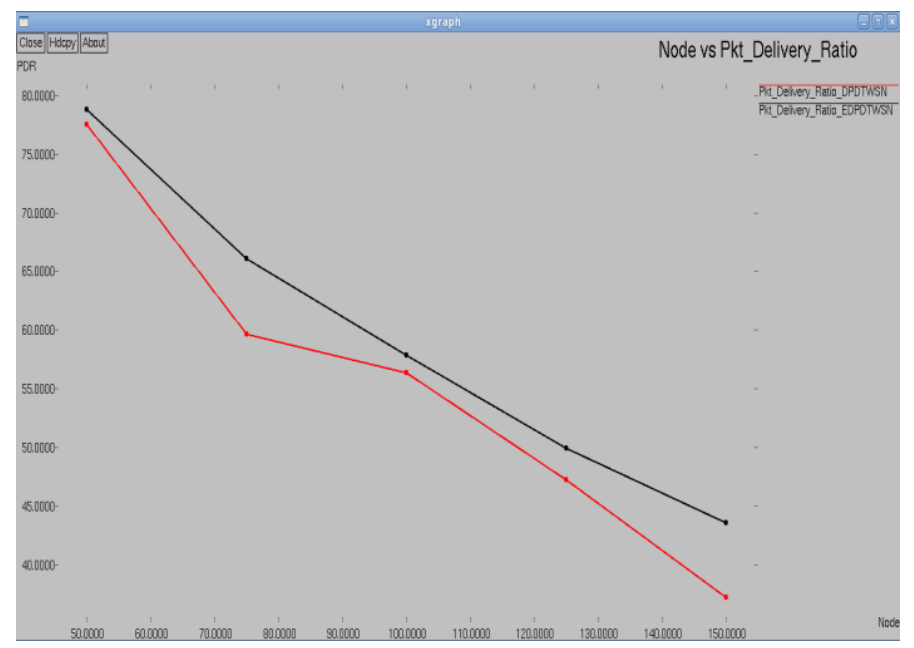

Fig 6: Graph Shows Node Vs Packet Delivery Ratio 


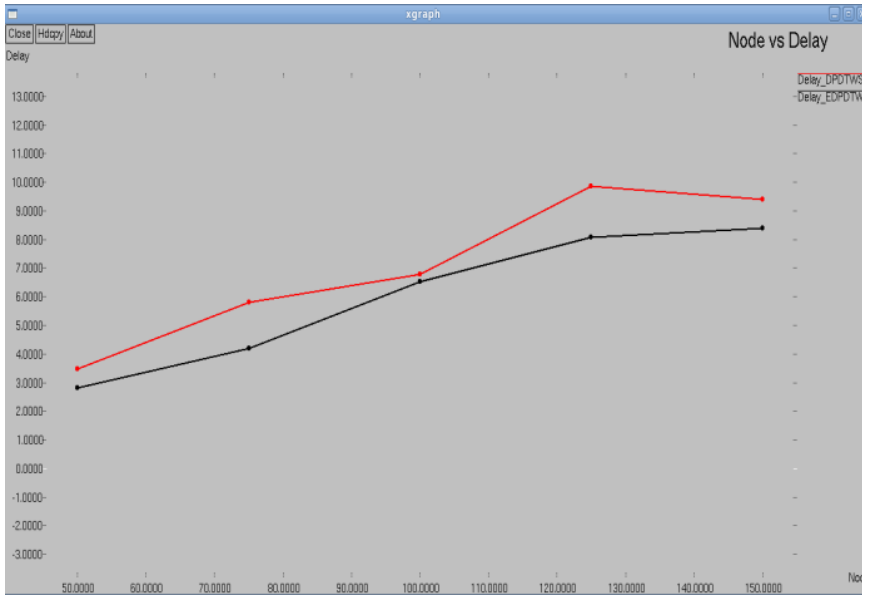

Fig 7: Graph Shows Node Vs Delay

Figure 7 shows that delay is in more in PAT as compared to EnOPATr system with different node scenario. The red line shows PAT system. Black line shows EnOPATr system.

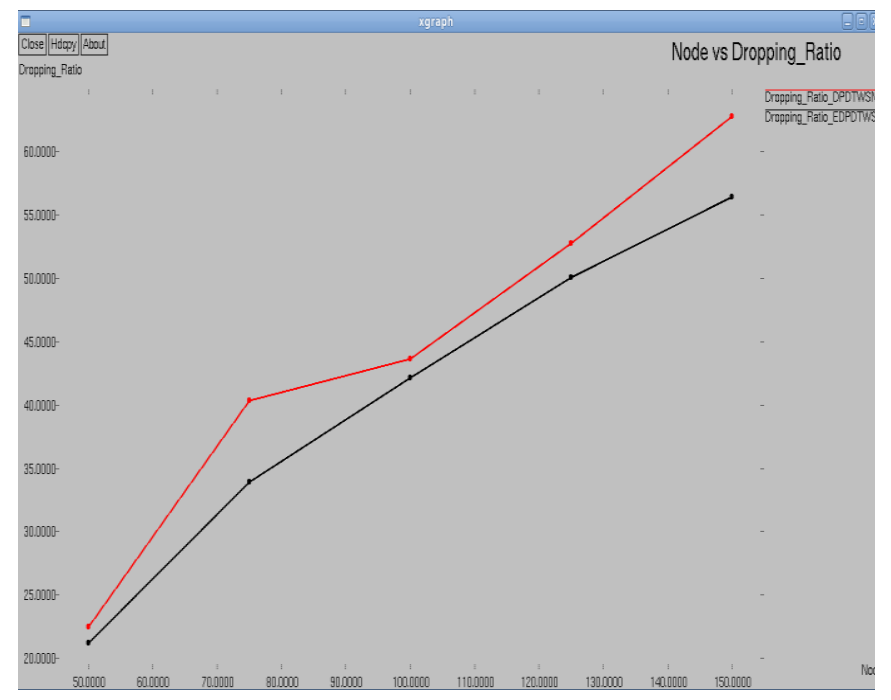

Fig 8: Graph Shows Node Vs Packet Dropping Ratio

Figure 8 shows that packet drop in ratio is more in PAT as compared to EnOPATr system with different node scenario. The red line shows PAT system. Black line shows ENOPATR system.

\section{C.Packet size}

Figure 9 shows that packet size vs. packet delivery ratio in ratio. EnOPATr shows more packet delivery ratio as compared to PAT system. The red line shows PAT system. Black line shows EnOPATr system.

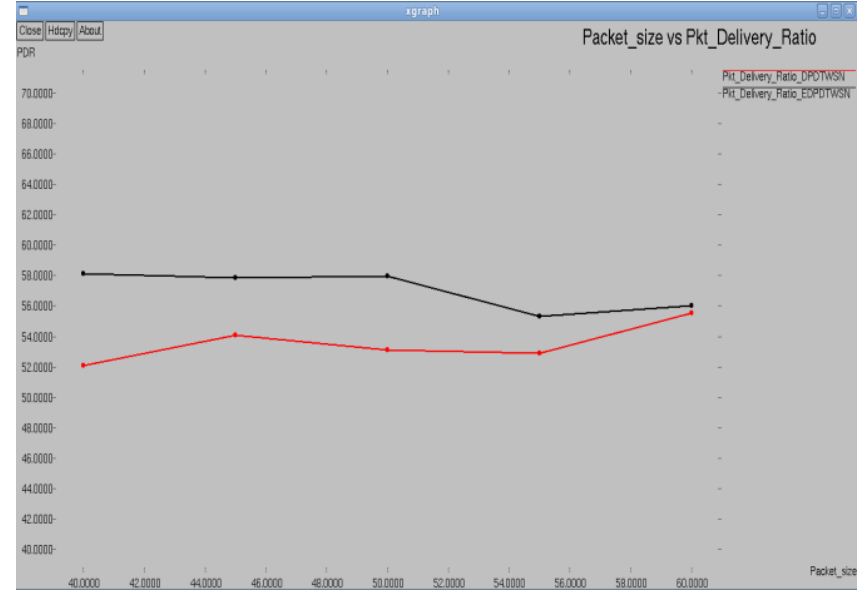

Fig 9: Graph Shows Packet Size Vs Packet Delivery Ratio

Figure 10 shows that packet size vs. delay in network. If more packets occur in network, the performance of PAT protocol is decreased as compared to EnOPATr. The EnOPATr system has lesser delay as compared to the traditional WSN network.

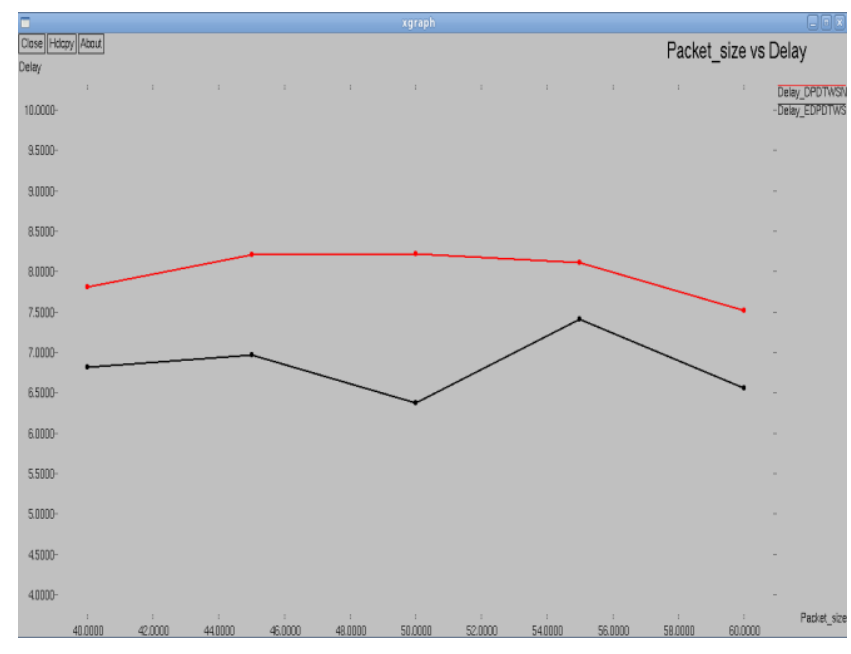

Fig 10: Graph Shows Packet Size Vs Delay

Figure 11 shows that packet size vs. packet dropping ratio in network. If more packets occur in network, the overall performance of EnOPATr protocol is improved as compared to PAT. The EnOPATr system has less packet drop as compared to the traditional WSN network. 


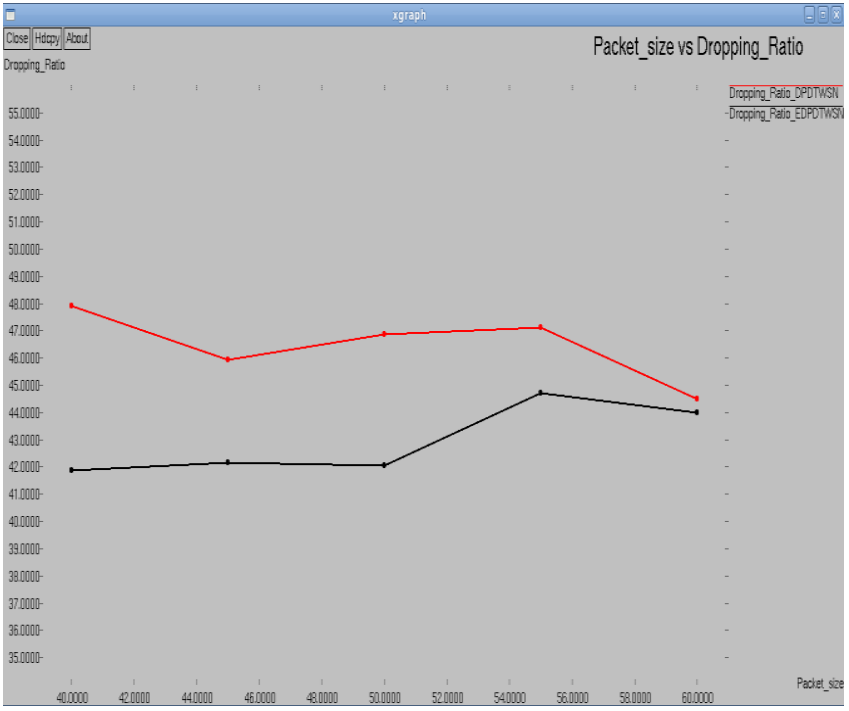

Fig 11: Graph Shows Packet Size Vs Packet Dropping Ratio

\section{Future Scope and Conclusion}

We can improve the existing system by adding Packet Discarding based on Node Clustering (PDNC) in EnOPATr. Within this paper, an innovative methodology consisting of EnOPATr system is proposed. The authors through result and discussion disc various advantages of the newly proposed system. It is necessary to undertake EnOPATr in different situations to consider both urgent and normal data for required processing.

\section{References}

[1] Khemapech, et al., "A survey of wireless sensor networks technology," in 6th Annual Post graduate Symposium on the Convergence of Telecommunications, Networking and Broadcasting, 2005

[2] O. B. A. Y. Sankarasubramaniam, and I. F. Akyidiz, ESRT: Eventto-sink reliable transport in wireless sensor networks. in Proceedings of ACM Mobihoc. 2003 , June 3

[3] S. S. a. D. Kumar, "An approach to optimize adaptive Routing Framework to provide QOS in Wireless Sensor Networks," in proceeding of International Journal of wireless Networks and Communication, vol. 1(1), pp. 55-69 2009

[4] Karanjawane, Ashwini D, Rohankar, Atul W, Mali, SD, Agarkar, AA, "Urgent Data Transmission in Wireless Sensor Network", International Journal of Innovative Research in Computer Science \& Technology (IJIRCST), ISSN:2347-5552,Volume-1, Issue2,November 2013

[5] Avinash Devare, G.K.Mohan, "Adaptive Data Transmission in WSN Using Enhanced Path Assured Transmission Protocol" Proceedings of the International Conference on Data Engineering and Communication Technology, Advances in Intelligent Systems and Computing 468, DOI 10.1007/978-981-10-1675-2_28,2017

[6] Sridevi, S, M. Usha, "Dynamic priority and fairness based congestion control framework for heterogenous wMSNs.", European Journal of Scientific Research 93, no. 4 (2012): 594-610.

[7] Tsai, Yao-Pin, Ming-Che Chung, Ru-Sheng Liu. "A forwarding mechanism for processing two categories of packets in wireless sensor networks." In. Proc. Computer Communication Control and Automation (3CA), International Symposium on, vol. 1, pp. 466469. IEEE, 2010.

[8] A.S. Devare , G.K. Mohan and Mayuri Gade "Transmitting Urgent Data Using ANKM Method" International Journal of Control Theory and Applications, Volume 9, Number 43, 2016

[9] Fukui, K, Wakamiya, N, Kawai, T., Murata, M., Oki Electric Industry Co., Ltd., 2010. Communication device and communication system. U.S. Patent 7,697,890..
[10] Xiong, Naixue, Zhao Wu, Yannong Huang, and Degang Xu. "Analyzing comprehensive QoS with security constraints for services composition applications in wireless sensor networks." Sensors 14 , no. 12 (2014): 22706-22736

[11] K. Ishibashi and M. Yano, a Proposal of Forwarding Method for Urgent Messages on a Ubiquitous Wireless Sensor Network. In Information and Symposium on. 2005, pp. 293-298.

[12] S. Sasirekha, S. Swamynathan, A Comparative Study and Analysis of Data Aggregation Techniques in WSN. Indian Journal of Science And Technology,2015 Oct.

[13] Jatinder Kaur, Gurjot Singh Gaba, Rajan Miglani, Ruchi Pasricha Energy Efficient and Reliable WSN based on Improved Leach-R Clustering Techniques.Indian Journal of Science and Technology,2015 July.

[14] Anandkumar, T., M. Vijayakumar. "Automatic Node Network Interface Using RF Module with Time Synchronization Technique." Wireless Communication 9, no. 2 (2017): 29-33. 